This item was submitted to Loughborough's Research Repository by the author.

Items in Figshare are protected by copyright, with all rights reserved, unless otherwise indicated.

\title{
Simple and robust framework for coupled aerothermal gas turbine simulation using low-mach and compressible industrial CFD solvers
}

PLEASE CITE THE PUBLISHED VERSION

http://dx.doi.org/10.2514/6.2016-1640

\section{PUBLISHER}

AIAA / @ The Authors

VERSION

AM (Accepted Manuscript)

\section{PUBLISHER STATEMENT}

This work is made available according to the conditions of the Creative Commons Attribution-NonCommercialNoDerivatives 4.0 International (CC BY-NC-ND 4.0) licence. Full details of this licence are available at: https://creativecommons.org/licenses/by-nc-nd/4.0/

\section{LICENCE}

CC BY-NC-ND 4.0

\section{REPOSITORY RECORD}

Legrenzi, Paolo, Karpaga V. Kannan, Gary J. Page, and Indi H. Tristanto. 2016. "Simple and Robust Framework for Coupled Aerothermal Gas Turbine Simulation Using Low-mach and Compressible Industrial CFD Solvers". figshare. https://hdl.handle.net/2134/22500. 


\title{
Simple and Robust Framework for Coupled Aerothermal Gas Turbine Simulation using Low-Mach and Compressible Industrial CFD Solvers
}

\author{
P.Legrenzi*, K.V.Kannan ${ }^{\dagger}$ and G.J.Page ${ }^{\ddagger}$ \\ Loughborough University, Loughborough, Leics, LE11 3TU, UK \\ I.Tristanto ${ }^{\S}$ \\ Rolls-Royce plc, P.O. Box 31 ,Derby, DE24 8BJ, UK
}

\begin{abstract}
Efforts are being made to achieve a coupled virtual aero-engine simulation using existing validated solvers. A methodology to easily couple a low-Mach number pressure-based code with a compressible density-based turbomachinery code for industrial application has been developed, based on boundary conditions update. Two techniques, namely, file based and memory based have been implemented to exchange data between the solvers in steady and unsteady RANS. Validation is carried out on a laminar flow for a convecting Taylor vortex test case. This approach has been applied to steady compressor/combustor and combustor/turbine simulations and extended to a simple unsteady simulation of a $2 \mathrm{D}$ Von Karman vortex shedding cylinder case and to a unsteady combustor/NGV/rotor test case. Results are shown, advantages and disadvantages of the two techniques are discussed.
\end{abstract}

\section{Introduction}

The industrial state-of-the-art for the design of a gas turbine generally treats each component in relative isolation, as a standalone part of a larger system, assuming it only weakly interacts with neighboring components through fixed boundary conditions. This approach has been the only one feasible for many years in the industry, for very practical reasons: computational cost and different modelling requirements. Nowadays, in the era of massive parallel computers, this limitation can be partially overcome and new strategies are to be developed to investigate the flow in a gas turbine. The interaction between different components and different physical processes needs to be taken into account, if a confident predictive simulation is to be achieved. Even though the increasing availability of computational resources and the improved efficiency of future flow solvers will allow the simulation of an entire engine, only with the use of multiple specialized flow solvers can the required accuracy and efficiency be obtained, taking into account the huge variety of flow phenomena that have to be simulated. This is particularly true when coupling turbomachinery parts and combustor. Moreover, industry requires the simulation to deliver results sufficiently accurate within an acceptable timeframe (typically an overnight run).

In combustors high swirl and free shear layer flows are dominant. A full LES simulation of the whole engine could be computationally prohibitive, but since it will greatly improve the prediction of detached flows and free turbulence in the combustor, ${ }^{1}$ this type of simulation may be performed in this portion of the engine only. The combustor is also characterised by heat release and chemical reactions, and additional transport equations have to be solved to model the combustion process. Again the solution of these equations can be limited to the combustor. The reaction is generally assumed to occur at constant pressure and the flow Mach number is usually very low. Hence pressure-based low-Mach number solvers are widely used

*Corresponding Author, Research Associate, AIAA Member, email: p.legrenzi@lboro.ac.uk

$\dagger$ Research Associate

¥Senior Lecturer, AIAA Senior Member

$\S$ Currently a Royal Society Industrial Fellow, AIAA Senior Member 
to model such flows. In the turbomachinery parts, a precise description of the turbulent boundary layers around the blades, tip gaps and leakage flows, is required. High Mach and Reynolds numbers characterise the flow. Hence density-based compressible flow solvers capable of handling rotating components and thin shear layers are generally used. In the industry the flow solvers for turbomachinery applications are based on RANS simulations, and years of experience led to a good degree of confidence in the various parameters needed to model the turbulence. A RANS or URANS approach, that is computationally affordable, may then be used in the turbomachinery parts.

A complete simulation of a virtual gas turbine can be achieved by coupling existing specialised flow solvers that has been developed through decades of experience in simulating each components. This type of approach, in which each zone is treated separately, is called zonal coupling and has previously been applied to gas-turbine simulation by some research groups. ${ }^{2-4}$

The aim of the work presented here is to develop a simple and reliable methodology to couple simulations of different components of a gas turbine, in order to properly understand their interactions and to be able to predict multi-components effects, such as combustion instabilities or combustor-turbine hot-streak migration. ${ }^{5}$ This is becoming more important as lean combustion is being introduced for aero-engines. Requirements for compactness and lightness in aeronautical applications make all the components strongly interacting; lean burn technology potentially increases combustor-turbine interactions. In that way in the future, simulations will be able to substitute, at least partially, very expensive experimental rig tests.

The main objectives are:

- To develop a framework for automated coupled simulation using industrial turbomachinery and combustor flow solvers via exchange of boundary conditions.

- To implement two methodologies, file based and memory based, to easily exchange data between the solvers

- To demonstrate the method for a steady RANS simulation of a realistic aero-engine geometry and extended the technique to unsteady simulations.

\section{Methodology}

The coupling procedure is based on boundary conditions exchange: the domains being coupled are simulated using their specific solver while exchanging boundary conditions data until convergence or at each time iteration. The coupling is essentially an outer iteration to update boundary condition. The inner iteration is the iterative process that each solver would perform to converge or partially converge the flow field for that given boundary condition. For steady state simulation the outer iteration is stopped when both solver and boundary condition updates reach a convergence. For unsteady iteration, the outer iteration reflects the physical time stepping. Data exchange and management of the coupling are fully automated; the file based coupling is driven by a Python application which exploits the interpolation capabilities of CHIMPS,${ }^{6}$ while the memory based coupling uses the open source coupler O-Palm. ${ }^{7}$ The choice of a coupler for the memory based coupling has the advantage of allowing an easy extension of the coupling to new software for future applications. The methodology is based on partially overlapping domains. ${ }^{8}$ Interpolation is carried out in each domain to provide new boundary condition data to the other domain.

The coupling involves two industrial validated codes, a low-Mach number pressure-based code for combustion and a density-based code for turbomachinery applications. Both use the finite volume discretisation but the first uses a cell center formulation while the latter uses a vertex based median dual control volume.

\section{A. Solvers}

\section{Low-Mach number Solver}

The solver uses a low-Mach number approximation to reduce the time stepping CFL restrictions. The assumption made is that for combustion applications the pressure in the combustion chamber has little variation and the flow is very slow compared to the speed of the acoustic waves. The code ${ }^{9}$ is pressure-based and uses a collocated cell-centered approach for unstructured grids. The variables are stored at control volume centers. Different first and second order accurate spatial schemes are available. All the second-order accurate schemes are implemented following a deferred correction scheme. ${ }^{10}$ The viscous dissipative term 
is evaluated using the method of Mathur and Murthy. ${ }^{11}$ The SIMPLE pressure correction approach for incompressible flows is adopted, and the method of Rhie and Chow ${ }^{12}$ is used to avoid pressure-velocity decoupling. In case of an unsteady simulation the transient term is calculated combining two implicit time schemes with a blending factor. The first-order Euler scheme and the second-order three level scheme are recovered when the default delimiting values for the blending factor are used. The code is parallelised using MPI.

\section{Compressible Solver}

The density-based code was designed to work on unstructured hybrid meshes. Data are stored at the cell vertices and dual control volumes are defined around each vertex, applying a vertex-centered approach.

The edge based data structure ${ }^{13}$ allows for a second order accurate spatial discretisation. The fluxes are split into an inviscid and viscous part. The inviscid flux discretisation is based on a flux-differencing scheme with a Jamesons type eigen scaling method, which combines the central differencing of the nonlinear inviscid flux with a smoothing flux based on one-dimensional characteristic variables. The viscous flux is approximated half-way along each edge, where the existing edge weights are used to approximate the flow variables gradients.

For a steady simulation the discrete equations are iterated towards the steady state with a time-marching procedure and local time stepping with a five stage Runge-Kutta scheme. ${ }^{14}$ A block-Jacobi preconditioner ${ }^{15}$ combined with a multigrid method are used to damp all the error modes and accelerate the convergence. An implicit dual time stepping ${ }^{16}$ is used for the unsteady solver. The code is fully parallel with high parallel performance. ${ }^{17}$ It exploits the OPlus parallel library. ${ }^{18}$

\section{B. Coupling procedure}

Both memory based and file based coupling mechanisms rely on data exchange through boundary conditions updates. Figure 1 shows a schematic representation of the exchange. The two domains are partially overlapping such that the inlet of the downstream domain is located in a region inside the upstream domain and the exit of the upstream domain is within the downstream domain. It should be noted that the two grids are generated independently from each other and the domains are simulated using independent solvers. Flow field data within an interpolation region of the upstream domain are interpolated into the boundary interface plane (inlet) of the downstream domain and vice versa.

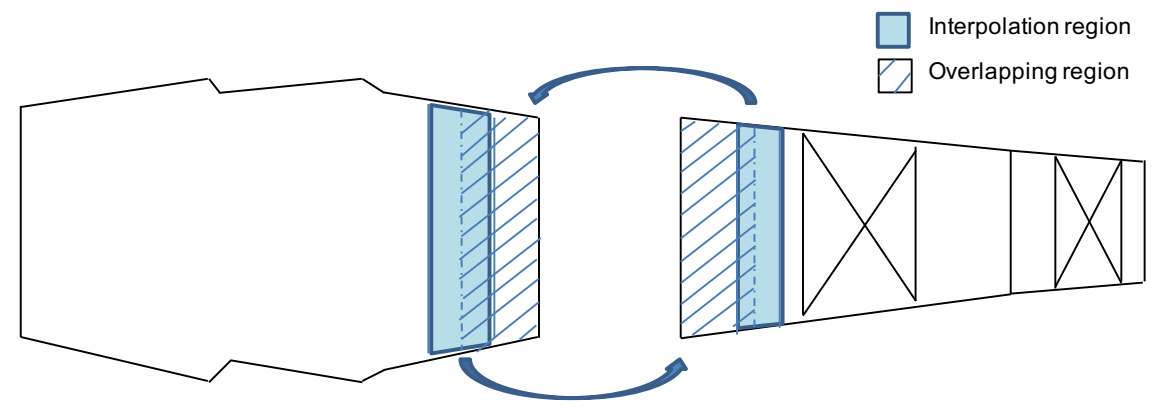

Figure 1. Coupling mechanism

\section{File based coupling}

For the file based coupling technique no modifications of the source codes are needed. The data for the interpolation are read from the existing flow files written by each specific solver using a common access library. The interpolation capability of CHIMPS is used to provide data on the boundary interface planes. The boundary data are written on files as a 2D profile. A Python script with a simple input file is used for the automated management of the whole coupling procedure. The sequence of the data exchange process can be either sequential or simultaneous. In sequential mode the codes run one after the other while in simultaneous the codes run concurrently. 


\section{Memory based coupling}

In the memory based coupling the two solvers have been modified such that the data can be directly exchanged through memory. In particular, using the coupler O-Palm and its interpolator CWIPI, each code is running in parallel and sharing the same MPI communicator. A driver is managing the coupling between the two (or more) applications. Other options are available in the O-Palm coupler to link different codes, which do allow a less intimate way of data exchange (via socket), but the direct introduction of a few routines in the source codes and the direct exchange through message passing was the preferred option to maximise the performance of the coupling. The approach exploited is a simultaneous rather than a sequential coupling. The idea is that running both the solvers together will be more computationally efficient once the space has been allocated on a cluster and a number of processes assigned to each solver.

\section{Interface conditions}

Low-Mach number and compressible codes solve two different set of equations: ${ }^{19}$ in the former the acoustics is completely removed and the pressure plays the role of a lagrangian multiplier to ensure continuity; the latter instead retains all the flow features while pressure is a thermodynamic variable linked to density through an equation of state. When coupling these two different physical models, attention has to be paid to ensure the interfaces do not produce unphysical behaviours. As an example acoustic waves should be allowed to freely leave the compressible domain at the interface boundary without encountering reflections.

The objective is that each domain should have a well-posed set of boundary conditions according to the physical nature of the problem. Only the necessary number of variables are imposed avoiding overspecification. Since some of the variables are free to adjust themselves to the flow and the solved equations are different, not all the desired quantities might be conserved at the interfaces.

A compromise has to be found in the interface region that allows for the two solutions to be consistent to a good degree, while conserving those physical quantities we are the most interested in for engineering applications. Massflow conservation is one of the first requirement in fluid machinery. In turbomachinery the stagnation pressure is also a key variable to describe the flow performance, but the different meanings of the pressure in the two solvers make its continuity at the interface somehow more problematic. In the end the energy of the fluid should be conserved: due to the different versions of the energy equation being solved and considering the variable engineers will actually be looking for, ensuring continuity of the temperature field seems to be a reasonable approach.

In the coupling procedure we aim at conserving the mass flow at the interface by making the local magnitude of momentum per unit volume continuum. If the local magnitude of momentum per unit volume $\rho|\mathbf{U}|$ has to be continuum at the interface a simple relation between density and velocities in the two domains can be drawn. In the low-Mach number and compressible domains holds respectively

$$
\rho^{L M}=\frac{P_{\text {therm }}}{R_{\text {gas }} T} \quad \rho^{C}=\frac{P}{R_{\text {gas }} T}
$$

where $P$ is the spatially and temporally variable pressure in the compressible domain and $P_{\text {therm }}$ the uniform reference thermodynamic pressure in the low-Mach number domain. From which, by imposing $\rho^{L M} U^{L M}=\rho^{C} U^{C}$ we obtain

$$
\frac{U^{L M}}{U^{C}}=\frac{\rho^{C}}{\rho^{L M}}=\frac{P}{P_{\text {therm }}}
$$

While conserving the massflow, in order to minimise the differences in density and velocities, the difference between pressure in the compressible domain $P$ and $P_{\text {therm }}$ should be always bounded in the region close to the interface. The coupling procedure is built such that $\frac{\left|P-P_{\text {therm }}\right|}{P_{\text {therm }}}$ is always verified to be small $(<1 \%)$ at the interface.

\section{Low-Mach number Solver (LMS) interfaces}

Dirichlet conditions are imposed. The variables at the interface boundaries are:

- LMS-INLET: Velocities $U_{x}, U_{y}, U_{z}$ and temperature $T$ profiles. To improve massflow conservation the massflow itself through the boundary might be imposed which involves the rescaling of the velocity profile accordingly 
- LMS-OUTLET: Gauge pressure $P_{g}$ profile (relative to reference thermodynamic pressure $P_{\text {therm }}$ )

\section{Compressible Solver (CS) interfaces}

The compressible solver uses a weak imposition of a characteristic type of boundary condition. This allows the acoustic waves normal to the boundaries to leave the domain without reflections. The weak imposition instead is a limiting factor for the accuracy of the interface. The variables at the interface boundaries are:

- CS-INLET: profile of $\rho|\mathbf{U}|$, flow angles and temperature $T$

- CS-OUTLET: Static pressure $P$ profile

For a steady case simulation a second alternative approach has been developed, based on pressure. While ensuring, as a reference pressure point, the average static pressure at the inlet interface to be equal to the thermodynamic reference pressure in the Low-Mach number domain, the inlet condition is modified as:

- CS-INLET2: profile of stagnation pressure $P_{0}$, flow angles and stagnation temperature $T_{0}$

which is a more common boundary condition in turbomachinery. In such configuration the massflow conservation is ensured by its imposition at the outlet plane of the domain (which can not be a coupling interface).

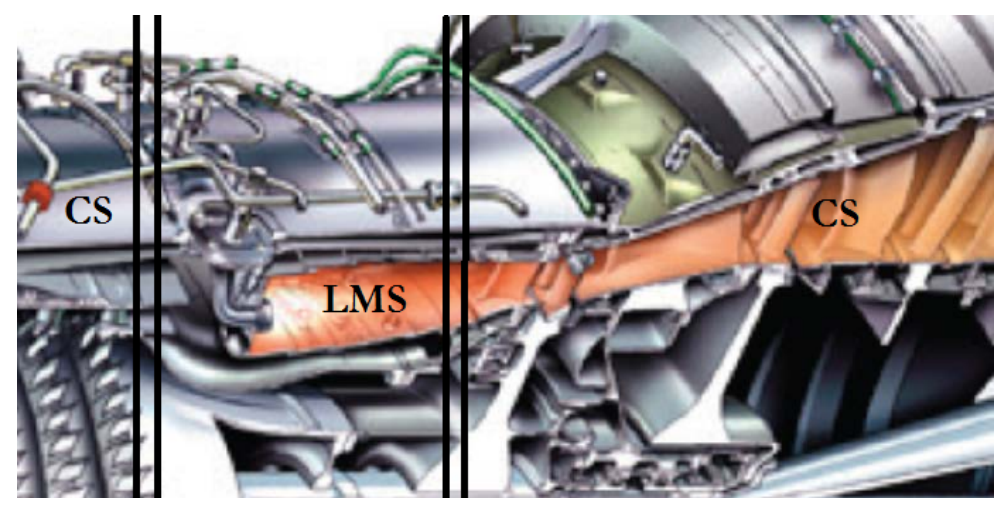

Figure 2. An example of compressor/combustor/turbine interfaces (CRolls-Royce

In a compressor/combustor coupled simulation, the compressible solver will be used for the upstream domain while the low-Mach number solver will be used for the downstream domain. At the combustor inlet the LMS-INLET condition will be specified while at the compressor outlet the CS-OUTLET will be used. In a combustor/turbine coupled simulation, the compressible solver with CS-INLET is used downstream and the low-Mach number solver with LMS-OUTLET is used upstream.

\section{Combustion}

For testing purposes of combustor-turbine interaction the EBU combustion model with a two-step reaction mechanism has been chosen. The variables mixture fraction, fuel mass fraction and carbon monoxide mass fraction are added to the solved equations in the Low-Mach number domain. Dealing with reacting flow in the combustor, the flow properties generally depends on species local concentration. At the moment the gas properties $\left(R_{\text {gas }}\right)$ in the turbomachinery code are constant and are chosen to be consistent with average properties at the interface plane.

\section{Validation of the method for Low-Mach/Compressible applications}

The method proposed above is here defined as Method A and it is tested on a simple case of a vortex convected from the low-Mach to the compressible domain and compared to other possible methods. For upstream low-Mach number and downstream compressible domains, Method A imposes $\rho U, \rho V, \rho W$ (or alternatively $\rho|\mathbf{U}|$ and flow angles) and $T$ in a weak formulation of the boundaries and using a characteristic 
treatment at the compressible inlet interface (CS-INLET). Other methods have been assessed for the inlet interface. Method B uses a stronger imposition at the boundary in which only the continuity equation is solved in the boundary control volume given the fluxes $\rho U, \rho V, \rho W$ interpolated from the low-Mach domain. Method B reads:

$$
\begin{gathered}
T^{C S}=T^{L M S} \quad ; \quad \rho^{C S}=\frac{P^{C S}}{R T^{L M S}} \quad ; \quad U^{C S}=\frac{(\rho U)^{L M S}}{\rho^{C S}} \\
V^{C S}=\frac{(\rho V)^{L M S}}{\rho^{C S}} ; \quad W^{C S}=\frac{(\rho W)^{L M S}}{\rho^{C S}}
\end{gathered}
$$

In this way the pressure (or density) is let free to fluctuate and a not over-imposed condition is obtained, but a stronger imposition is used and no characteristic treatment is adopted. Method C and Method D instead are treating the inlet boundary as a pure interface and an over-imposed condition is proposed. All variables are strongly imposed on the boundary node. Method $\mathrm{C}$ obtains the density from a reconstructed low-Mach number pressure $P^{L M S}=P_{\text {ref }}+P_{g}$

$$
\begin{gathered}
T^{C S}=T^{L M S} ; \rho^{C S}=\frac{P^{L M S}}{R T^{L M S}} ; U^{C S}=\frac{(\rho U)^{L M S}}{\rho^{C S}} \\
V^{C S}=\frac{(\rho V)^{L M S}}{\rho^{C S}} ; \quad W^{C S}=\frac{(\rho W)^{L M S}}{\rho^{C S}}
\end{gathered}
$$

while Method D involve the direct injection of all variables

$$
\begin{gathered}
T^{C S}=T^{L M S} \quad ; \quad \rho^{C S}=\frac{P_{r e f}}{R T^{L M S}}=\rho^{L M S} \quad ; \quad U^{C S}=\frac{(\rho U)^{L M S}}{\rho^{C S}}=U^{L M S} \\
V^{C S}=\frac{(\rho V)^{L M S}}{\rho^{C S}}=V^{L M S} \quad ; \quad W^{C S}=\frac{(\rho W)^{L M S}}{\rho^{C S}}=W^{L M S}
\end{gathered}
$$

In all the proposed methods the variables that are object of interpolation between the two domains are $\rho U, \rho V, \rho W, T$ and $P_{g}$. As a consequence there might be a difference in Method D between the values of velocities $U^{L M S}$ obtained from interpolated $\left((\rho U)^{L M S}\right)$ and what would be their directly interpolated values. It is still considered to be better to achieve mass conservation to always interpolate and try to conserve $\rho U$, $\rho V$ and $\rho W$.

In the following the methods are applied and tested on a vortex convection problem, and further applied to a cylinder shedding problem and a downstream square obstacle test case.

\section{A. Taylor Vortex}

The case was proposed by Peet \& Lele ${ }^{21}$ and consists of a Taylor Vortex ${ }^{22}$ crossing the low-Mach/compressible interface. The purely tangential velocity disturbance is initialised as:

$$
u_{t}=\frac{\mathcal{M} r}{16 \pi \nu^{2} t^{2}} \exp \left(-\frac{r^{2}}{4 \nu t}\right)
$$

The initial time $t$ is set so that the Reynolds number based on the initial radius of maximum velocity and the main convective velocity $\bar{U}$ is $R e=\frac{\bar{U} r_{M a x}}{\nu}=330$. The Mach number based on the convective velocity is 0.15 . The angular momentum $\mathcal{M}$ is chosen to set the maximum velocity disturbance $U_{M A X}^{\prime}$ to $1 \%$ of the mean convective velocity $\bar{U}$. The grid of each domain uses a uniform spacing of 200 by 200 , with 2 elements used in the normal direction together with periodic conditions to simulate a quasi two dimensional field.

All methods have shown good agreement of velocity field in the overlapping region (the maximum error being of the order of $2 \%$ of the maximum initial disturbance). The vertical velocity field is shown in Fig. 3 together with the vorticity magnitude at three different times during the crossing of the vortex. The tangential (vertical) velocity along the horizontal line passing through the center of the vortex, when the vortex is located inside the overlapping region, is shown in Fig. 4 for Method A. As can be seen the agreement of the velocity field is good. Looking at the vorticity field some inconsistencies are found in the shape of 
LMS

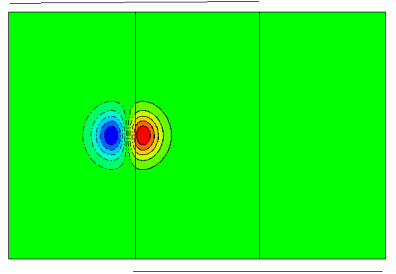

CS
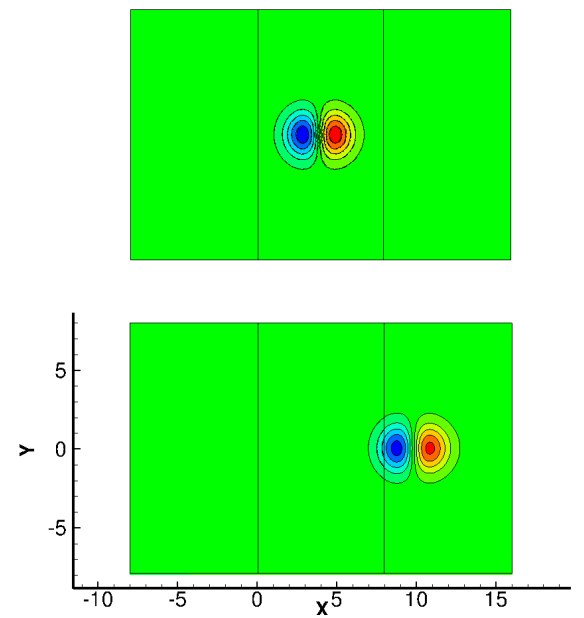

LMS

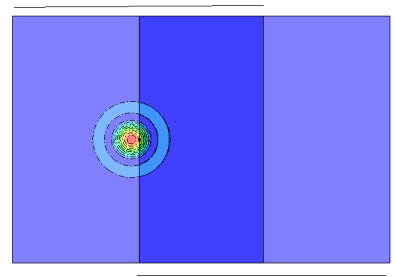

CS
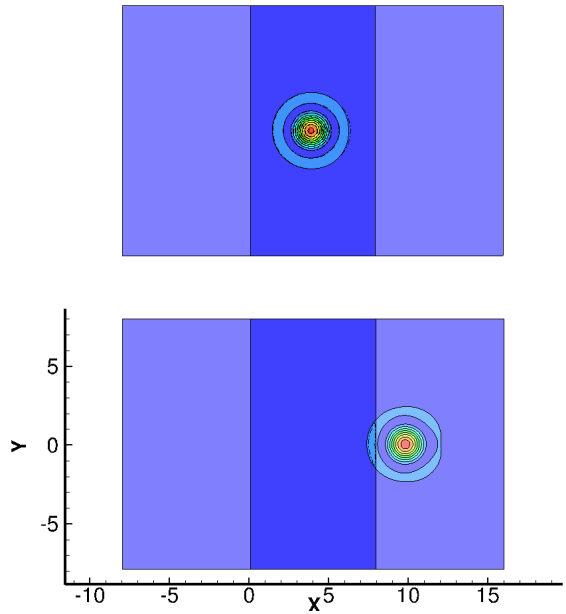

Figure 3. Vertical Velocity (left) and Vorticity Magnitude (right), Taylor vortex crossing the interface from Low-Mach to compressible

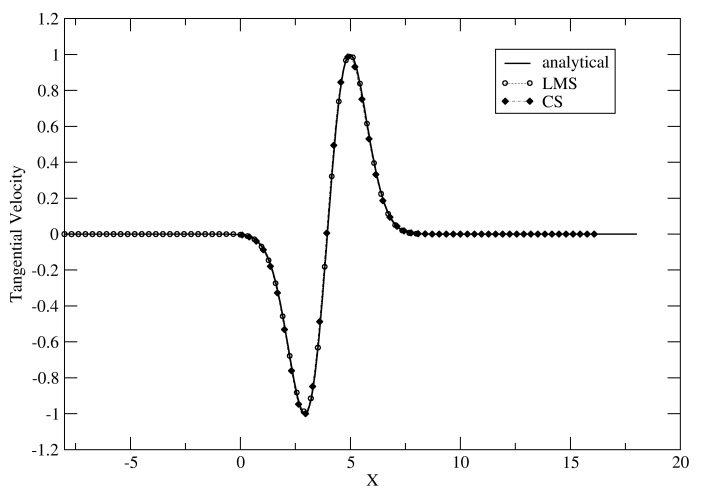

(a)

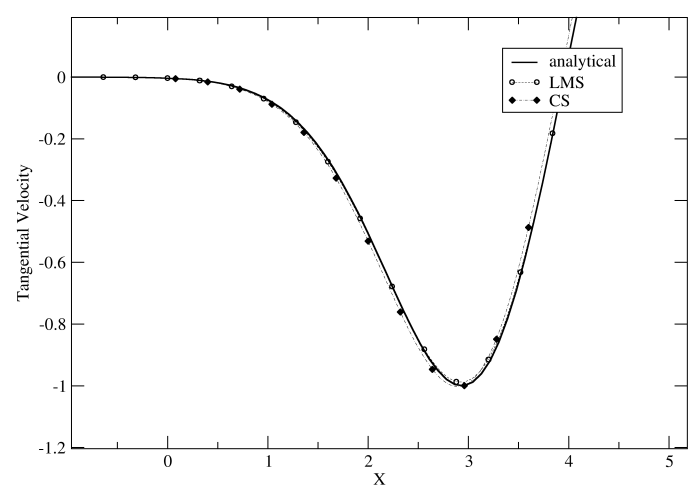

(b) detail

Figure 4. Normalised Tangential Velocity along the vortex axis, vortex located inside the overlapping region 


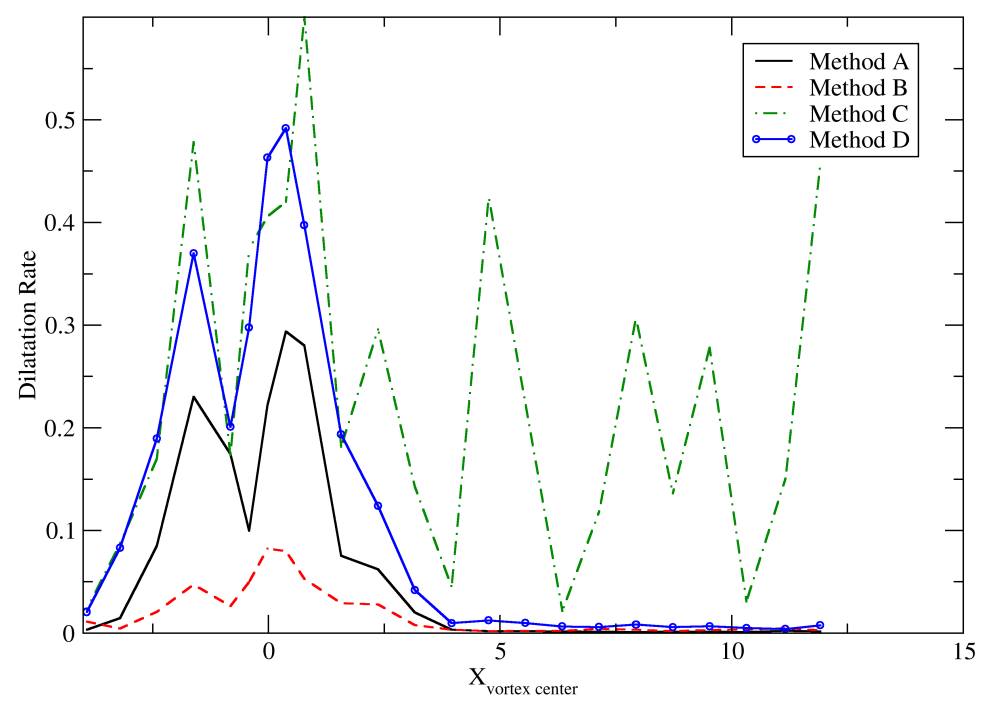

Figure 5. Maximum Rate of Dilatation obtained in the compressible domain against vortex center position with different CS-inlet treatments, Taylor vortex

the vortex that has left the overlapping domain, possibly due to the velocity gradients not guaranteed to be continuous at the interface.

The Taylor vortex is an incompressible vortex and it is by definition divergence free. Following the analysis proposed in Peet \& Lele ${ }^{21}$ as the disturbance introduced is small compared to the convective field, the vortex should not introduce dilatation in the compressible field. The maximum value of the rate of dilatation found throughout the whole compressible domain is plotted against the axial coordinate position of the center of the vortex as it moves through the domain in Fig. 5.

The methods which showed best performance are Method A and Method B. In both methods, after the initial peak encountered when the vortex is crossing the interface the maximum dilatation rate returns to the initial state once the vortex has fully entered the domain. Method D has shown greater values of dilatation rate as well as higher levels remaining after the vortex has crossed. Method C instead never recovers the initial values of dilatation rate and peaks are found when the vortex has already left the interface. By looking in Fig. 6(a) at the distribution of the dilatation rate in the compressible domain (in Fig. 6 is shown the downstream domain when the vortex has almost completely left the upstream domain) we note how Method A and B show a similar wavy behaviour, Method D concentrates its peaks only in the region of the inlet interface, while Method $\mathrm{C}$ shows the worst behaviour. When the vortex crosses the inlet interface of the compressible domain a density fluctuation is generated, coupled to the pressure disturbance introduced. As Method D tries to keep the density constant at the inlet, this method is the one which behaves the best in terms of having a smooth rounded field of density (Fig.6(b)). In Method A and B pressure is more free to fluctuate and density does so as well, showing a more wavy behaviour. It should be noted that the density fluctuation introduced for this test case are very small, less than $0.001 \%$.

A major difference with respect to the method proposed by Peet stands in the low-Mach number interface boundary treatment. In our approach solely the value of gauge pressure $P_{g}$ is imposed at the exit boundary, while $u, v, w$, were imposed together with a Neumann condition for the pressure (ensuring continuity of velocities and gradients of pressure) in Peet \& Lele. ${ }^{21}$ This work proposes a weaker imposition of downstream flow characteristics to the upstream flow field, as in realistic engine test cases the imposition of velocity components at the low-Mach number domain exit is not suitable to ensure a well behaved simulation in terms of mass conservation between inlet and outlet and requirement on the velocity field divergence characteristics. Following the asymptotic analysis of Muller ${ }^{19}$ the gauge pressure corresponds to the second order pressure in the Mach number expansion and it is not coupled with density and temperature. The leading error term 


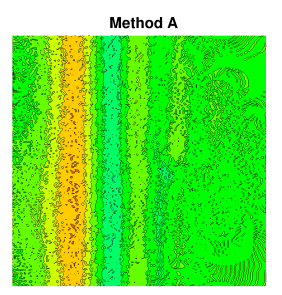

Method C

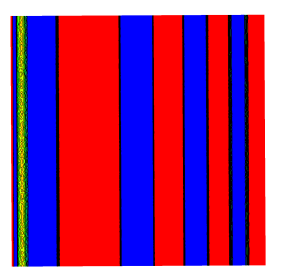

(a) Rate of dilatation

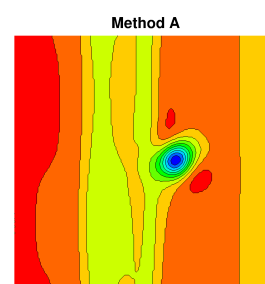

Method C

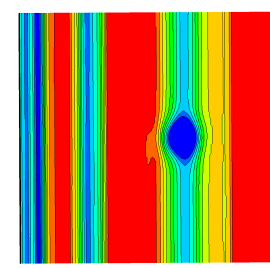

(b) Density field

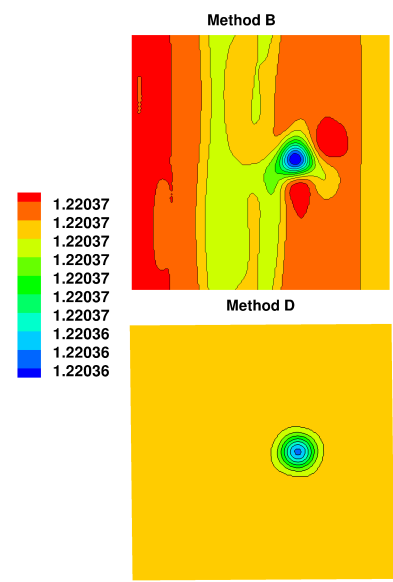

Figure 6. Compressible domain when the vortex has left the low-Mach domain

in the pressure definition scales with the square of the Mach number. The use of Dirichlet conditions for this variable allows for a direct comparison between pressures in the two domains:

$$
P^{L M S}=P_{r e f}+P_{g}
$$

When comparing the pressures in the two domains a difference up to $170 \%$ of the initial disturbance $\left(\rho\left(U_{M A X}^{\prime}\right)^{2}\right)$ is found, which is concentrated at the boundary interfaces. This number is within the expected errors. In fact following Peet \& Lele, ${ }^{21} 170 \%$ of the $1 \%$ disturbance introduced is $1.7 \%$ of the mean flow field, which is of the order of $M^{2}=0.0225$. In Fig. 7 is shown the maximum value of normalised pressure difference as the vortex crosses the interface (vertical lines are showing the overlapping region limits, the abscissa is the vortex center position).

\section{B. Cylinder Shedding}

To extend the vortex simulation to multiple vortices crossing the low-Mach number/compressible interface a circular cylinder has been used in the upstream domain to shed vortical structures downstream. The coupling procedure described in Method A has been applied to an unsteady case of a cylinder shedding at $R e=100$. Results are shown in figure 8(a) and 9 for velocity magnitude and pressure. The results has been successfully compared to single code standalone simulations. A snapshot from standalone compressible and low-mach simulations is reported in figure $8(\mathrm{~b})$ and $8(\mathrm{c})$. As can be seen from figure 8(a) the interface generates some small inconsistencies in the overlapping region, mainly due to the weak imposition of the interface conditions in the compressible domain, to the time step difference between the the two boundary conditions (the single time step is converged separately by the two solvers) and to the inherent differences in the numerical discretisation schemes of the two codes. The comparison of figure 8 (a) with the full standalone low-Mach simulation of figure 8(b) shows how the shape of the vortices crossing the interface is maintained in the downstream compressible domain. Figure 8(c) instead shows some differences, confirming the two codes are not expected to deliver exactly the same flow field. It is clearly the upstream domain with the cylinder that determines the vortex shedding characteristics and flow field downstream. The obtained Strouhal number is $S t=0.167$ for all cases, matching experimental data.

\section{Downstream Obstacle}

The vortex test case (and the circular cylinder) is characterised by a disturbance travelling from the upstream to the downstream domain. In order to assess the proposed Methods in a case representative of a downstream potential field affecting the upstream flow (blade potential field in a real engine) a square cylinder case was designed. The obstruction square object is located in the compressible domain just two axial grid cells downstream of the exit of the low-Mach number domain. The flow is such that $R e=100$ and $M=0.15$. 


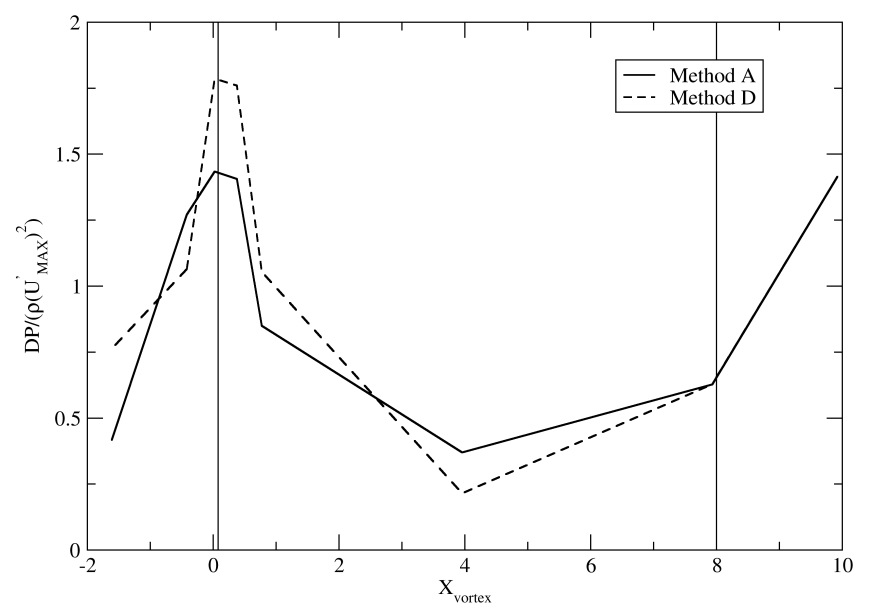

Figure 7. Difference in pressure between the two domains for Method A and D as the vortex crosses the interface region, Taylor vortex

Method A and Method C are compared in Fig. 10. As can be seen Method D has shown to be fully reflective: as expected the inlet interface, conserving density and temperature from the low-Mach domain, tries to keep the value of pressure constant and equal to $P_{r e f}$, which is un-physical for compressible flows and produces reflections.

The conclusion of this validation is that Method A is the preferred among the assessed methods for its good performance in the vortex crossing test case and because of its intrinsic non reflective treatment. Method B has shown very similar behaviour to Method A. Method D is found to be reflective in the square cylinder test case. Method $\mathrm{C}$ has shown the worst results.

\section{Applications}

The coupling procedure has been applied to a steady simulation of real compressor/combustor and combustor/turbine interactions and it has been extended to an unsteady 2D cylinder vortex shedding and unsteady combustor/NGV/rotor case.

\section{A. Compressible/Low-Mach coupling}

Further simulations were carried out on the coupling procedure based on file exchange for steady RANS simulation of a real compressor-combustor coupled simulation explained in detail in the work of Kannan \& Page $^{20}$ (2014). The region of interest is shown in Fig. 11. Figure 12 and Fig. 13 show the slice of normalised velocity magnitude at the interface of the Outlet Guide Vane (OGV)/pre-diffuser components. The dark solid contour lines represent the simulation in the turbomachinery components simulated using the compressible flow solver while the dotted contour lines represents the simulation in the combustor components simulated using the low-Mach number flow solver. The contours without any lines depict the simulation carried out by the compressible flow solver using low-Mach number preconditioning. The two vertical lines represent the interface regions. The values at the interface match very well. This confirms the accurate interpolation and transfer of boundary conditions in the coupling process. Though only the values at the boundaries are explicitly exchanged, the gradients at the interface are also transferred smoothly from one domain to another. Since the coupling is carried out in both directions, the effects of the downstream combustor components on the upstream turbomachinery components are also seen in the figure. The downstream combustor simulation alters the velocity and pressure field in the OGV and this can be observed when comparing with the full compressible code simulation. The influence of the downstream component is observed up to $20 \%$ of the OGV chord distance from the OGV trailing edge towards upstream. Upstream of $20 \%$ of the OGV chord, 


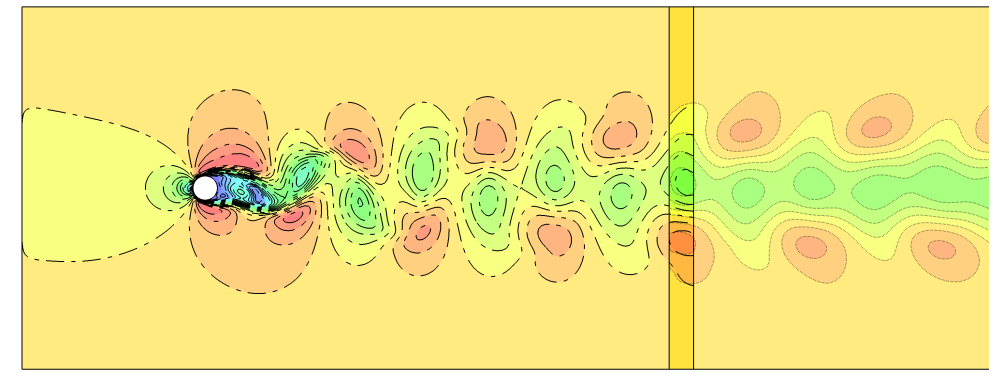

(a) Low-Mach (dashed-dotted)/compressible (dotted) coupling, Velocity magnitude $V_{\text {mag }}$
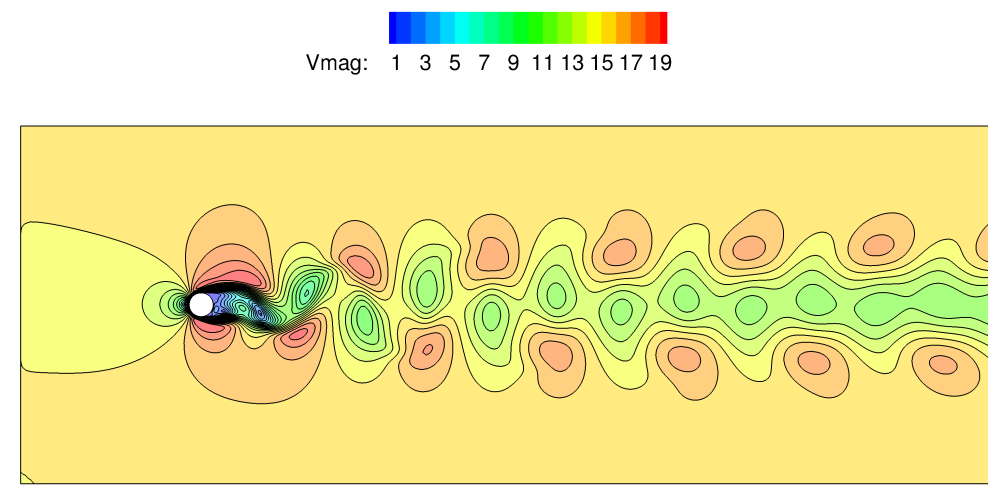

(b) Standalone low-Mach simulation, Velocity magnitude $V_{\text {mag }}$

Vmag: $\begin{array}{lllllll}1 & 3 & 5 & 7 & 9 & 11 & 13151719\end{array}$

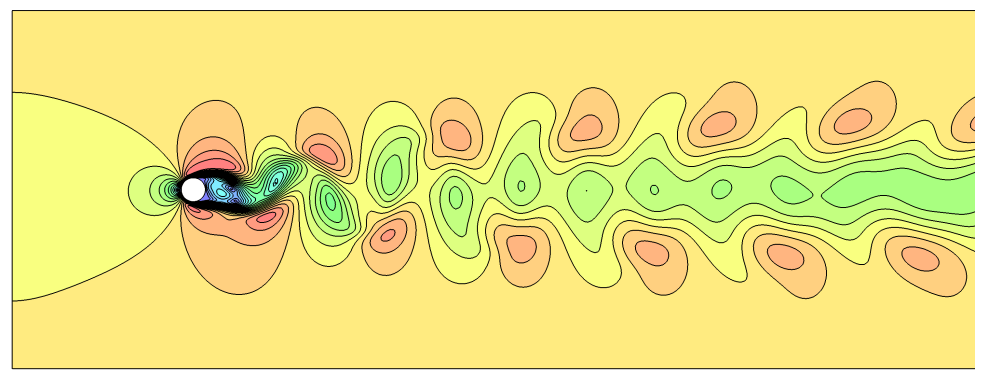

(c) Standalone compressible simulation, Velocity magnitude $V_{m a g}$

Figure 8. Snapshot of $2 \mathrm{D}$ cylinder shedding at $\mathrm{Re}=100$ 
P $\quad \begin{array}{lllllllll}-50 & -37.5 & -25 & -12.5 & 0 & 12.5 & 25 & 37.5 & 50\end{array}$

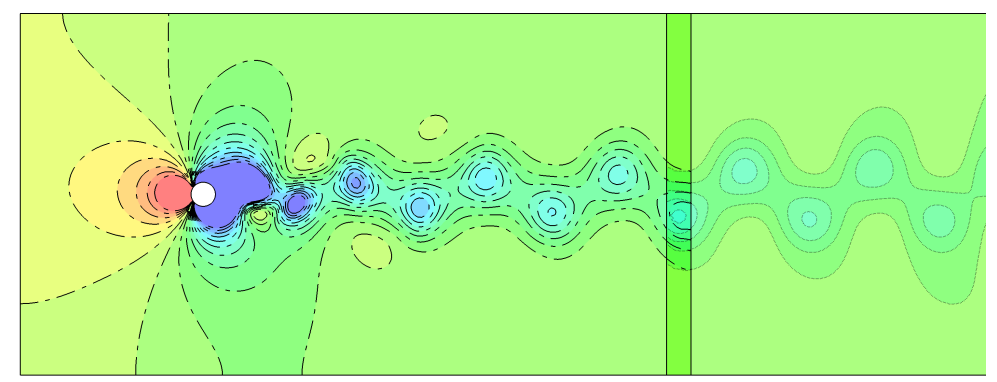

Figure 9. Low-Mach (dashed-dotted)/compressible (dotted) coupling, Gauge pressure $P_{g}$

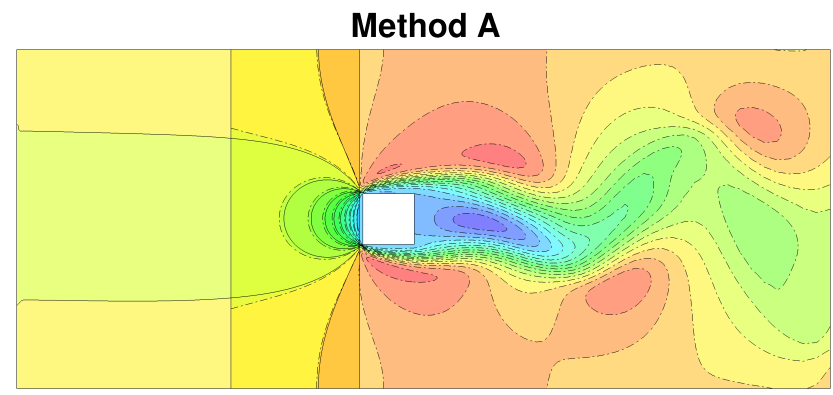

Method D
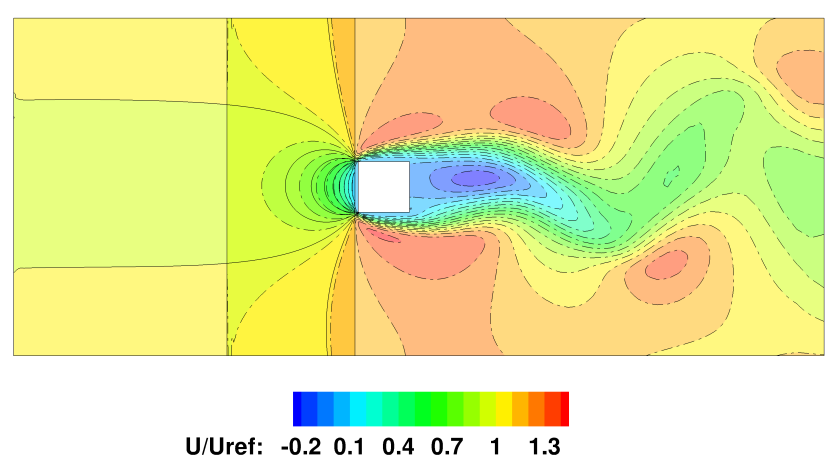

Figure 10. Assessment of Method A and D for downstream disturbance, low-Mach (solid line)/compressible (dashed-dotted) coupling, square cylinder 
the effect is negligible.

Figure 14 shows the exit planes of IGV, rotor, OGV and inlet of injector along with the mid-OGV radial slice of the coupled simulation results for the turbomachinery and combustor components. The wakes from the OGVs are accurately captured by the downstream low-Mach number code. Since modern lean burn injectors are larger, the wakes from the OGVs play an important role in deciding the quality of the injector inlet condition. From the figure, nearly eight out of ten wakes from the OGV enter the injector inlet region. From the axial slice at the injector inlet, the imprint of the OGV wakes is also observed. The observed injector condition is obtained from the two way coupling of the upstream and downstream components and this level of detail would not have been possible if the data from the coupling technique had not been implemented.

Table 1 gives the coupling time statistics. The computing cost for the entire process is shown. The computing was carried out on the HPC Midlands Hera supercomputer. The full KIAI rig simulation and the coupled simulation are carried out using 168 cores with a total of 14 nodes. It is seen that the coupled simulation of the full experimental setup is found to $37 \%$ quicker when compared to a simulation using just the compressible flow solver for the whole domain. This reduction in the computational time is mainly due to the application of the appropriate use of codes designed for their applications in the coupling process. The lowMach number combustor code was found to be quicker in computing the flow in the isothermal combustor geometry when compared to the simulation using the compressible turbomachinery code employing low Mach number preconditioning. The code is also faster to converge highly swirling flow compared to the turbomachinery code. These differences resulted in an overall reduction in the coupled full rig simulation time when compared to the simulation of the full rig using the compressible turbomachinery code. Thus by employing the coupling technique using appropriate flow solvers for the turbomachinery and combustor geometries, the best of all the codes are exploited. The method is currently being extended for unsteady RANS simulation for the same geometry and the results are presented in Kannan \& Page ${ }^{24}$ Kannan. ${ }^{25}$

\section{B. Low-Mach/Compressible coupling}

The geometry consists of a simplified real rich-burn combustor, two passage Nozzle Guide Vane (NGV) and one rotor blade. The Low-Mach number solver results simulates the combustor while the compressibile solver is used for the turbine. The coupling interfaces and the overlapping region are located between the combustor and NGV and they can be seen in figure 15 as two vertical lines at the combustor exit, while a mixing plane approach is used between NGV and rotor. The EBU combustion model is used in the combustor to simulate the turbulent reaction of Jet-A fuel. The $k-\epsilon$ turbulence model is used in both solvers for a steady computation.

In figure 15(a) and figure 15(b) are shown an isosurface plot of normalised temperature and a slice of the normalised velocity magnitude field respectively. As can be seen from the picture the flow is smoothly crossing the interface and the flow features are captured at the interface. The influence of the presence of the blades at the combustor exit is clearly seen in figure 15(b). More details on the obtained results are shown in Legrenzi et al. ${ }^{23}$

The same geometry of combustor/turbine used for steady simulation has been used for an unsteady run. The rotor pitch has been adjusted so to obtain periodic conditions with a blade count of 2 NGVs/4 rotors. A sliding plane approach is used for the rotor. In Fig. 16 the normalised temperature and normalised axial velocity are shown.

\section{Future work}

The variable "pressure" has shown to be the most difficult to make consistent betweeen the two domains (see vortex test case), because of its different meanings in the low-Mach number and compressible approximations. When dealing with low-Mach number domain usptream and compressible domain downstream, the Dirichlet exit boundary condition for pressure (LMS-OUTLET) has occasionally shown instability problems ans it is not a well posed condition to be used together with massflow inlet in the low-Mach number domain. Furthermore we are aware acoustic perturbations are fed into the low-Mach number domain where they cannot be supported by the incompressible governing equations. For these reasons we are investigating

the possibility of using a body force as proposed by Stanford University ${ }^{2}$ to be applied in the overlapping region of the low-Mach number domain. The body force drives the upstream solution to the downstream 
desired value. In this way the pressure field will be completely uncoupled between the two domains. In fact a convective outflow is used at the outlet of the low-Mach number domain instead of pressure exit. Initial results on the square cylinder test case are promising and application to a real test case will follow.

\section{Conclusions}

A methodology to easily couple a low-Mach number pressure-based combustor code with a compressible density-based turbomachinery code has been developed using file and memory based techniques. It has been successfully applied to real aero-engine simulations. The method has also been demonstrated for unsteady cases. The file based coupled simulation of compressor/combustor was found to be computationally quicker by $37 \%$ when compared to the uncoupled simulation of the same case using the compressible solver for the entire domain. This reduction in the computational time is mainly due to the application of the appropriate use of codes designed for their applications in the coupling process. The coupling procedure using different specialised solvers has demonstrated to be an efficient way to carry out multi-components gas turbine simulations.

\section{Acknowledgments}

The support by COPA-GT Marie Curie FP7, Rolls-Royce PLC and HPC-Midlands is gratefully acknowledged. The project is partially funded by UK TSB SILOET (Strategic Investment in Low Carbon Engine Technology). The third author is partially funded by The Royal Society through Industry Fellowship programme for his work in this research. The authors thanks Dr. M. Dianat for the initial setup of the file based coupling infrastructure. Technical support from Drs C. Nastase, M. Annand and M. Staufer at Rolls-Royce are also gratefully acknowledge by the authors.

\section{References}

\footnotetext{
${ }^{1}$ Gicquel, L.Y.M. and Staelbach, G. and Poinsot, T., Large Eddy Simulations of gaseous flames in gas turbine combustion chambers, Progress in Energy and Combustion Science, 38, 2012

${ }^{2}$ Schluter, J. U. and Wu, X. and Kim, S. and Shankaran, S. and Alonso, J. J. and Pitsch, H., A Framework for Coupling Reynolds-Averaged With Large-Eddy Simulations for Gas Turbine Applications, ASME Journal of Fluids Engineering, Vol 127, 2005

${ }^{3}$ Turner, M. G. and Norris, A. and Park, B. and Veres, J. P, High-Fidelity Three-Dimensional Simulation of the GE90, AIAA 2003-3996, 2004

${ }^{4}$ Insinna, M. and Salvadori, S. and Martelli, F., Simulation of Combustor/NGV interaction using coupled RANS solvers: validation and application to a realistic test case ASME Turbo Expo 2014: Turbine Technical Conference and Exposition, Dusseldorf, Germany, 2014.

${ }^{5}$ Dorney, D. J. and Gundy-Burlet, K. L and Sondak D. L., A Survey Of Hot Streak Experiments And Simulations, International Journal of Turbo \& Jet-Engines, Vol 16, 1999

${ }^{6}$ Alonso, J. J., CHIMPS: A High-Performance Scalable Module for Multi-Physics Simulations AIAA 2006-5274, Joint Propulsion Conference \& Exhibit, Sacramento, California, 2006

${ }^{7}$ Piacentini, A. and Morel, T. and Thevenin, A. and Duchaine, F., O-Palm: an Open Source Dynamic Parallel Coupler V International Conference on Computational Methods for Coupled Problems in Science and Engineering, 2011

${ }^{8}$ Collado Morata, E., Impact of unsteady aerothermal environment on the turbine blades temperature PhD thesis, Universite de Toulouse, Oct 2012.

${ }^{9}$ Anand, M. S. and Eggels, R. and Staufer, M. and Zedda, M. and Zhu, J., an advanced unstructured-grid finite-volume design system for gas turbine combustion analysis, Proceedings of ASME 2013 Gas Turbine India Conference, Bangalore, India, 2013

${ }^{10}$ Ferziger, J. H. and Peric, M.,Computational Methods for Fluid Dynamics, Springer, 2002

${ }^{11}$ Mathur, S. R. and Murthy, J. Y., A Pressure-Based Method for Unstructured Meshes, Numerical Heat Transfer, Vol 31, 1997

${ }^{12}$ Rhie, C. M. and Chow, W. L., A numerical study of the Turbulent Flow Past an Isolated Airfoil with Trailing Edge Separation, AIAA Journal, Vol 21, Num 11, 1983

${ }^{13}$ Crumpton, P. I. and Moinier, P. and Giles, M. B., An Unstructured Algorithm for High Reynolds Number Flows on Highly-Stretched Grids, 10th Int. Conf. Num. Meth. for Laminar and Turbulent Flows, Swansea, England, 1998

${ }^{14}$ Martinelli, L., Calculations of Viscous Flows with a Multigrid Method, PhD thesis, Dept of Mech and Aerospace Eng, Princeton University, USA, 1987

${ }^{15}$ Moinier, P., Algorithm Developments for an Unstructured Viscous Flow Solver, PhD thesis, University of Oxford, UK, 1999

${ }^{16}$ Crumpton, P. I. and Giles, M. B., Implicit time accurate solutions on unstructured dynamic grids, AIAA 95-1671, 1995
} 
${ }^{17}$ Hills, N., Achieving high parallel performance for an unstructured unsteady turbomachinery CFD code, The Aeronautical Journal, Num 3100, 2007

${ }^{18}$ Crumpton, P. I. and Giles M. B., Multigrid aircraft computations using the OPlus parallel library, Parallel Computational Fluid Dynamics, Implementations and Results using Parallel Computers, Elsevier Science, 1995

${ }^{19}$ Muller, B., Low-Mach-number asymptotics of the Navier-Stokes equations, Journal of Engineering Mathematics, 1998

${ }^{20}$ Kannan, K. V., Page, G. J., Coupling of Compressible Turbomachinery and Incompressible Combustor Flow Solvers for Aerothermal Applications ASME GT2014-26118, 2014

${ }^{21}$ Peet, Y. V., Lele, S. K., Computational Framework for Coupling Compressible and Low Mach Number Codes AIAA Journal, Vol. 26, No. 8, August 2008

${ }^{22}$ Taylor, G. I., On the Dissipation of Eddies Aeronautical Research Council, Reports and Memorandum No. 598, 1918

${ }^{23}$ Legrenzi, P. and Page, G. J. and Tristanto, I., An integrated Simulation of a Combustor/Hp Turbine Stage Using Coupled Industrial CFD Solvers ASME TurboExpo, June 13-17, Seoul, Korea, 2016

${ }^{24}$ Kannan, K. V. and Page, G. J., Automated Multi-code URANS Simulation of Compressor-Combustor Components ASME TurboExpo, June 13-17, Seoul, Korea, 2016

${ }^{25}$ Kannan, K.V., Coupling of Compressible Turbomachinery and Incompressible Combustor Flow Solvers for Gas Turbine Applications, PhD thesis, Loughborough University, Loughborough, UK, 2015 


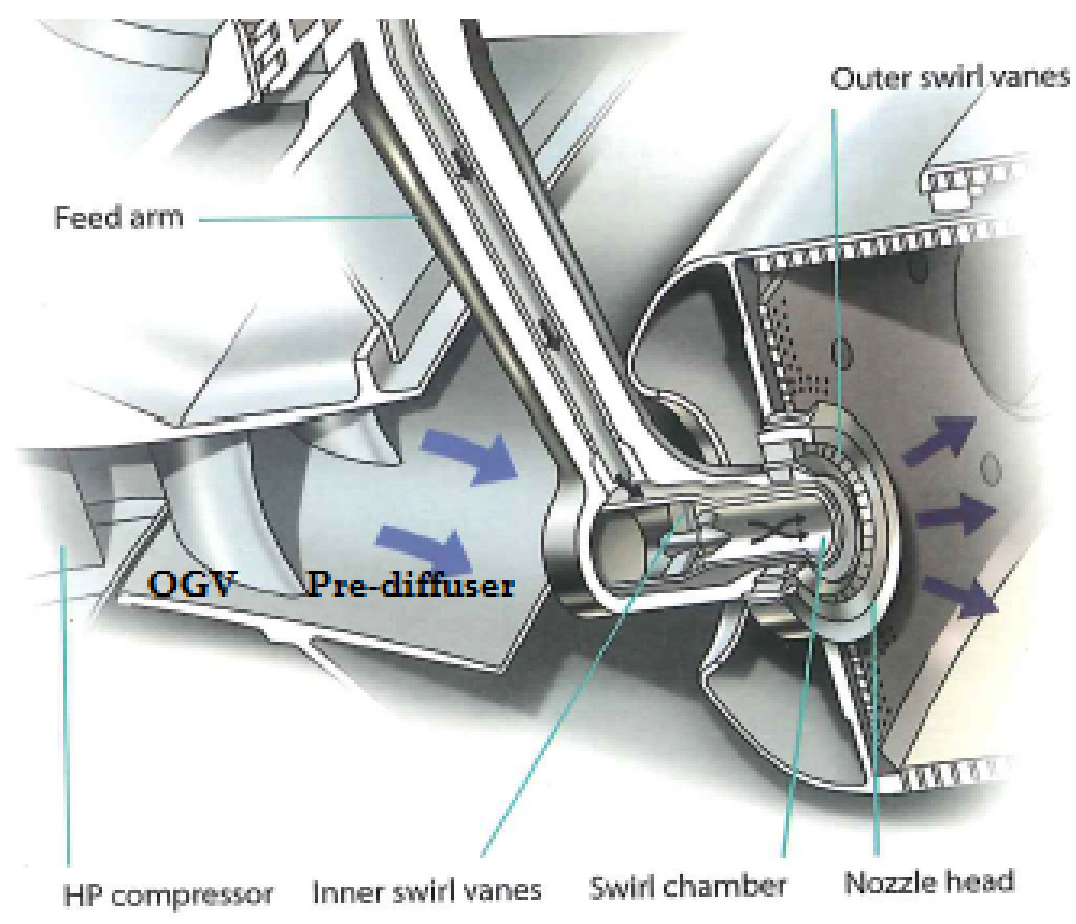

Figure 11. OGV/pre-diffuser interface for a modern injector (c)Rolls-Royce

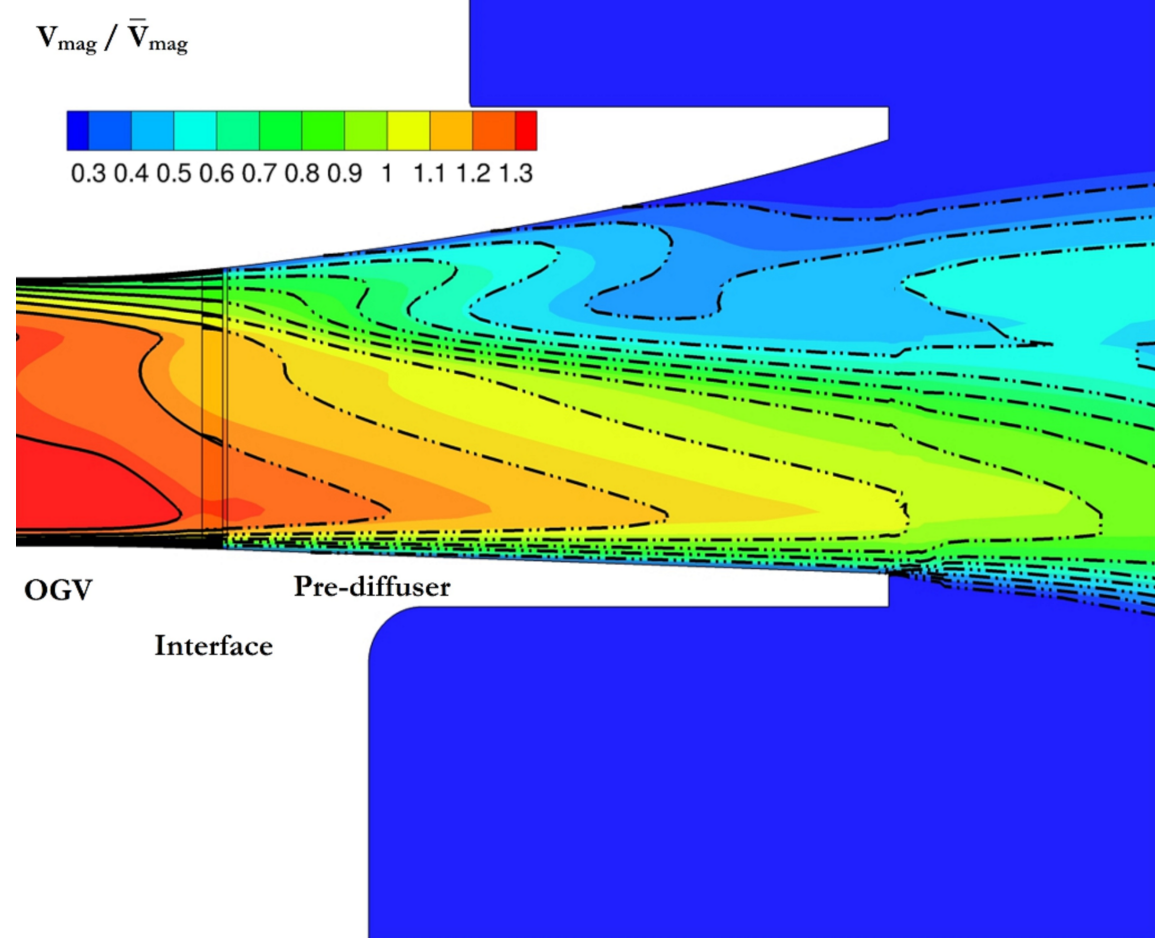

Figure 12. OGV/pre-diffuser interface for coupled vs uncoupled simulation ${ }^{20}$ 


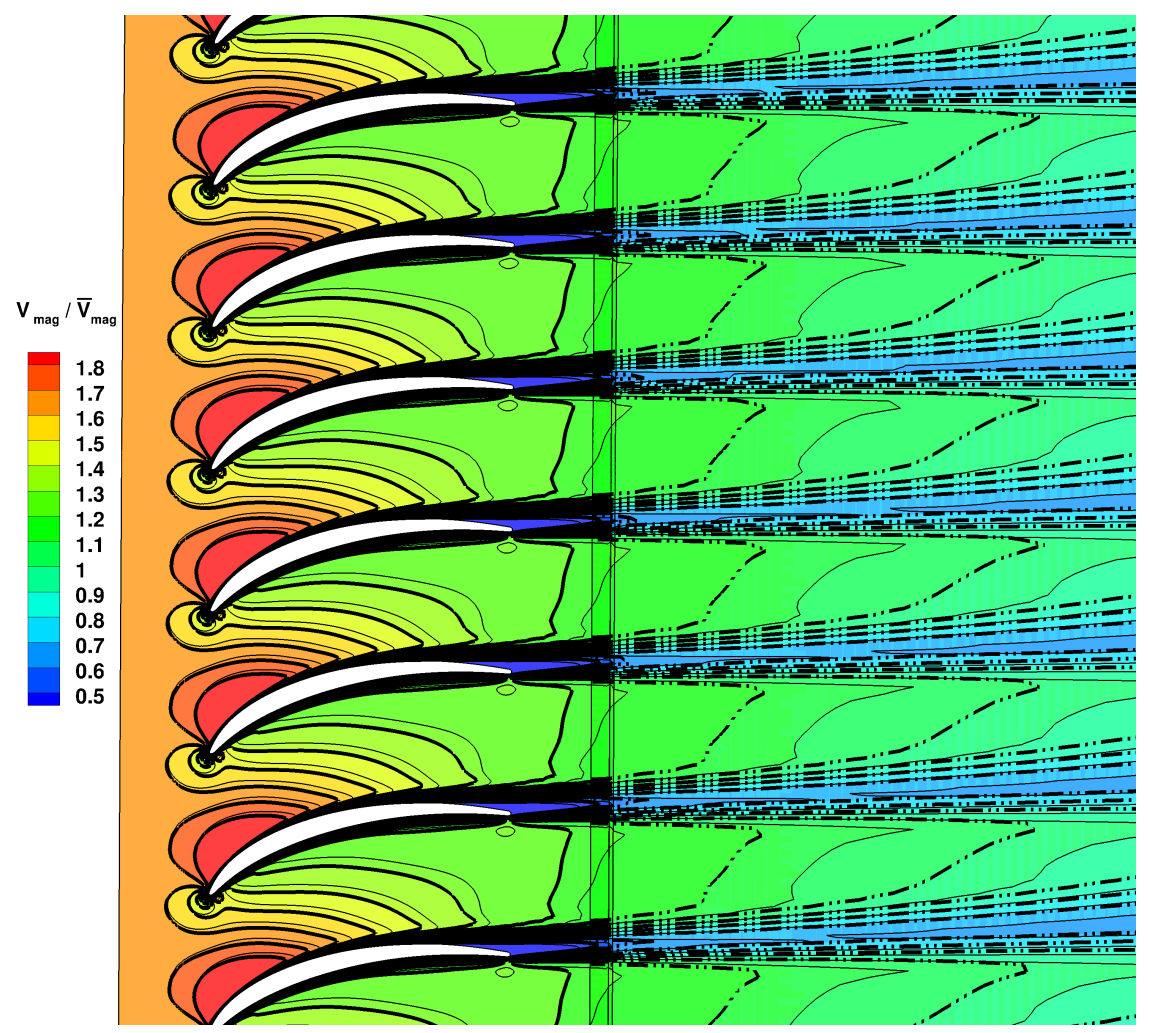

Figure 13. Wakes at $\mathrm{OGV} /$ pre-diffuser interface for coupled vs uncoupled simulation

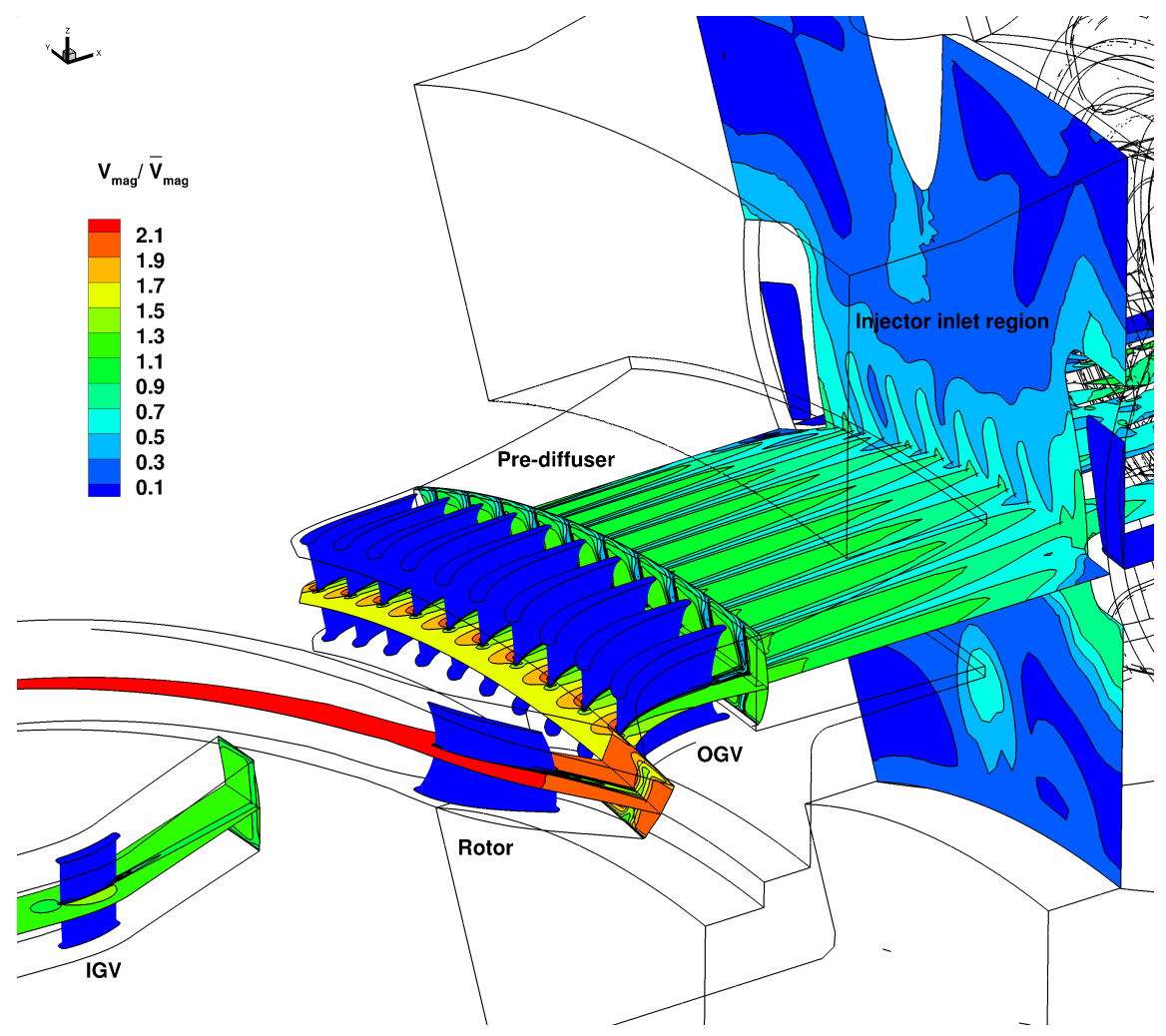

Figure 14. Radial and vertical cut planes of normalised velocity magnitude for turbomachinery/combustor coupled simulation 
Table 1. Comparative Time statistics of coupling operation using the same computing resources

\begin{tabular}{|c|c|c|c|c|c|c|c|}
\hline Operation & $\begin{array}{l}\text { Wall } \\
\text { time per } \\
\text { inner } \\
\text { iteration } \\
\text { (s) }\end{array}$ & Iterations & $\begin{array}{l}\text { Wall time } \\
\text { per outer } \\
\text { iteration } \\
\text { (s) }\end{array}$ & $\begin{array}{l}\text { Outer } \\
\text { It- } \\
\text { era- } \\
\text { tions }\end{array}$ & $\begin{array}{l}\text { Total wall } \\
\text { time }(\mathrm{s})\end{array}$ & $\begin{array}{l}\text { Total } \\
\text { wall } \\
\text { time } \\
\text { (hrs) }\end{array}$ & $\begin{array}{l}\text { Share } \\
\text { of total } \\
\text { coupled } \\
\text { simulation }\end{array}$ \\
\hline 1.CS Initial & 4.9 & 1500 & - & - & 7350 & 2 & $11.67 \%$ \\
\hline 2.LMS Initial & 3.2 & 1300 & - & - & 4160 & 1.16 & $6.77 \%$ \\
\hline 3.CS simulation & 4.9 & 600 & 2940 & 9 & 726,460 & 7.35 & $42.58 \%$ \\
\hline 4.LMS simulation & 3.2 & 600 & 1920 & 9 & 17,280 & 4.8 & $28 \%$ \\
\hline $\begin{array}{l}\text { 5.CS I/O file opera- } \\
\text { tion }\end{array}$ & - & - & 230 & 9 & 2070 & 0.58 & $3.4 \%$ \\
\hline 6.LMS I/O operation & - & - & 41.3 & 9 & 371.7 & 0.1 & $0.6 \%$ \\
\hline 7.CS to LMS coupling & - & - & 31 & 9 & 279 & 0.08 & $0.5 \%$ \\
\hline 8.LMS to CS coupling & - & - & 450 & 9 & 4050 & 1.125 & $6.48 \%$ \\
\hline $\begin{array}{l}\text { Total Coupled simu- } \\
\text { lation }\end{array}$ & - & - & - & - & 62,020 & 17.145 & $100 \%$ \\
\hline $\begin{array}{l}\text { Full KIAI Rig Simu- } \\
\text { lation }\end{array}$ & 39 & 2,500 & - & - & 97,730 & 27.15 & $157.5 \%$ \\
\hline
\end{tabular}




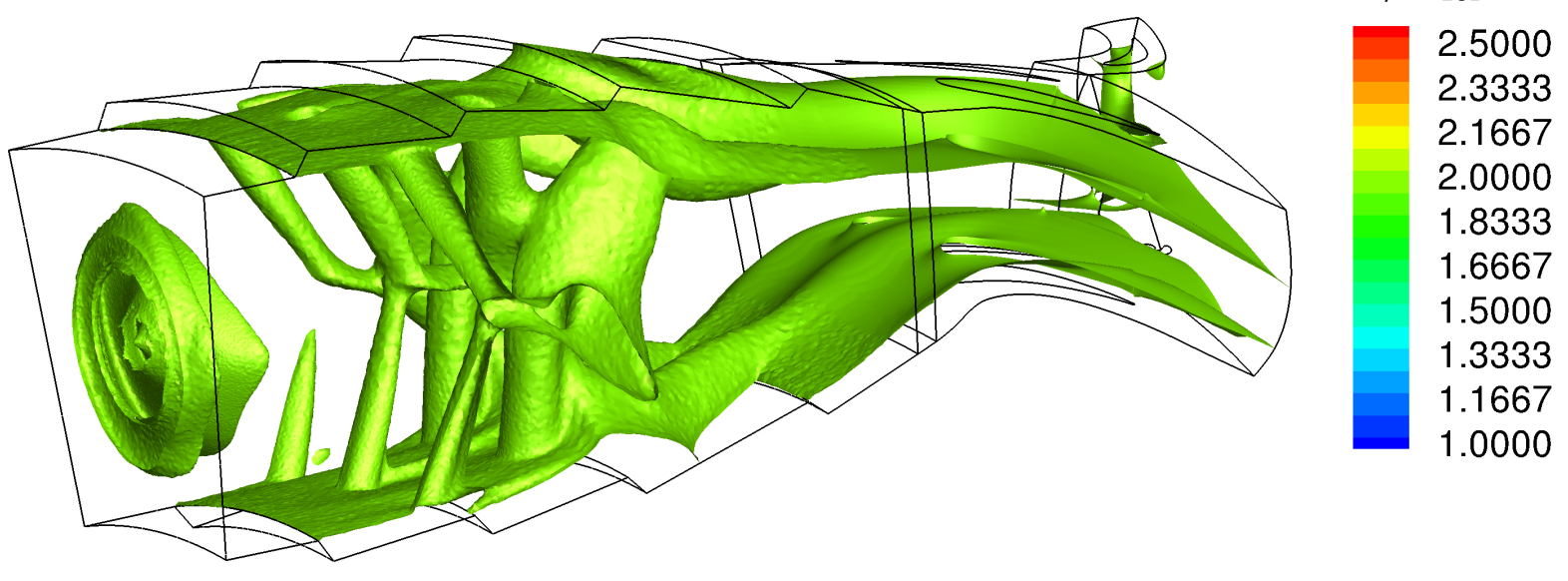

(a) Normalised temperature $T / T_{\text {ref }}=2$

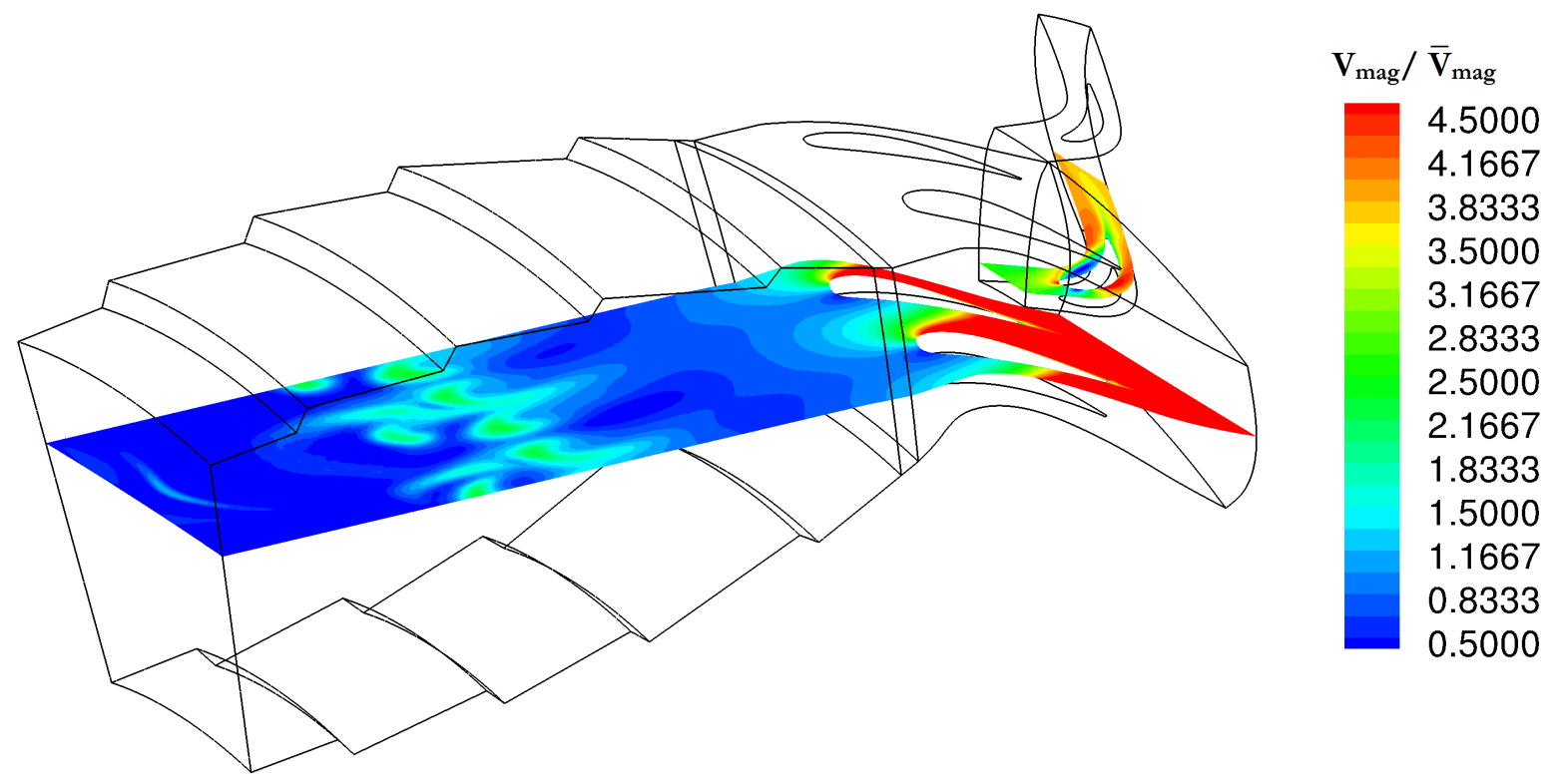

(b) Normalised velocity magnitude $V_{\text {mag }} / \bar{V}_{\text {mag }}$

Figure 15. Low-Mach/compressible coupling, combustor-turbine interaction 


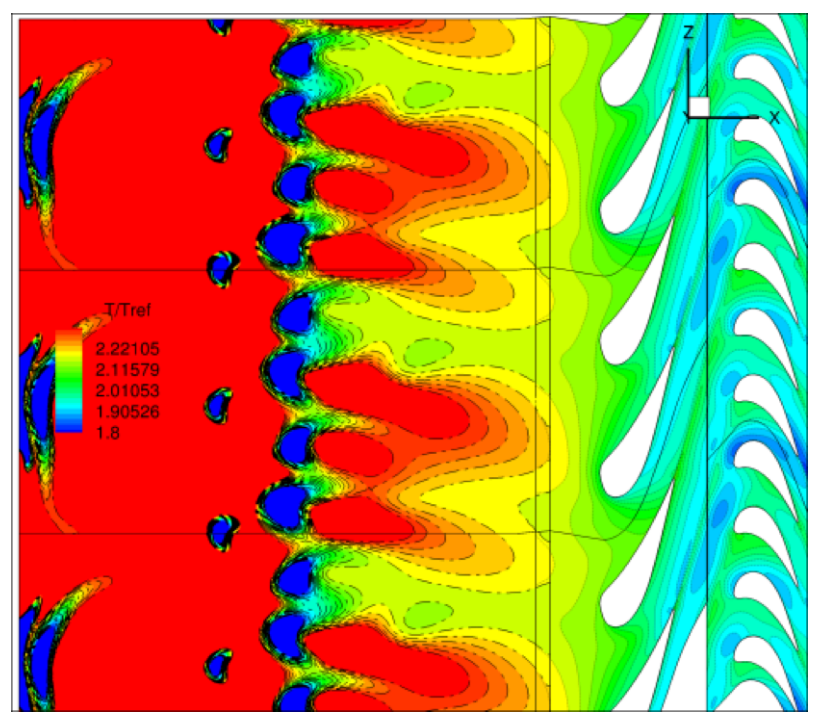

(a) Normalised Temperature

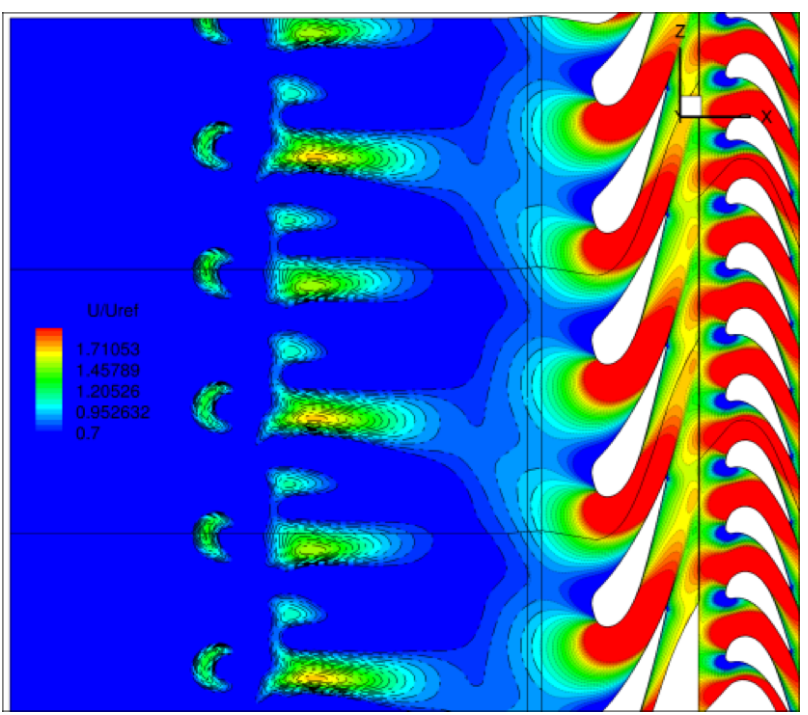

(b) Normalised Axial Velocity

Figure 16. Low-Mach (dashed-dotted)/compressible (dotted) coupling, Combustor/NGV/Rotor unsteady snapshot 